\title{
Towards an explanation of transverse single-spin asymmetries in proton-proton collisions: the role of fragmentation in collinear factorization
}

\author{
Koichi Kanazawa, ${ }^{1,2}$ Yuji Koike, ${ }^{3}$ Andreas Metz, ${ }^{2}$ and Daniel Pitonyak ${ }^{4}$ \\ ${ }^{1}$ Graduate School of Science and Technology, Niigata University, Ikarashi, Niigata 950-2181, Japan \\ ${ }^{2}$ Department of Physics, Barton Hall, Temple University, Philadelphia, Pennsylvania 19122, USA \\ ${ }^{3}$ Department of Physics, Niigata University, Ikarashi, Niigata 950-2181, Japan \\ ${ }^{4}$ RIKEN BNL Research Center, Brookhaven National Laboratory, Upton, New York 11973, USA
}

\begin{abstract}
We study the transverse single-spin asymmetry for single-hadron production in proton-proton collisions within the framework of collinear twist-3 factorization in Quantum Chromodynamics. By taking into account the contribution due to parton fragmentation we obtain a very good description of all high transverse-momentum data for neutral and charged pion production from the Relativistic Heavy Ion Collider. Our study may provide the crucial step towards a final solution to the longstanding problem of what causes transverse single-spin asymmetries in hadronic collisions within Quantum Chromodynamics. We show for the first time that it is possible to simultaneously describe spin/azimuthal asymmetries in proton-proton collisions, semi-inclusive deep-inelastic scattering, and electron-positron annihilation by using collinear twist-3 factorization in the first process along with transverse momentum dependent functions extracted from the latter two reactions.
\end{abstract}

PACS numbers: 12.38.-t, 12.38.Bx, 12.39.St, 13.75.Cs, 13.88.+e

Introduction The field of transverse single-spin asymmetries (SSAs) in hard semi-inclusive processes began some four decades ago with the observation of the large transverse polarization (up to about $30 \%$ ) of neutral $\Lambda$ hyperons in the process $p B e \rightarrow \Lambda^{\uparrow} X$ at FermiLab [1]. People noticed early on that the naïve collinear parton model cannot generate such large effects [2]. It was then pointed out that SSAs for single-particle production in hadronic collisions are genuine twist-3 observables for which, in particular, collinear 3-parton correlations have to be taken into account in order to have a proper description within Quantum Chromodynamics (QCD) 3]. This formalism later on was worked out in more detail and also successfully applied to SSAs in processes like hadron production in proton-proton collisions, $p^{\uparrow} p \rightarrow h X-$ see, e.g., Refs. [4 10]. Here we focus on SSAs in such reactions, which were extensively investigated in fixed target and in collider experiments.

Let us now look at the generic structure of the spindependent cross section for $A\left(P, \vec{S}_{\perp}\right)+B\left(P^{\prime}\right) \rightarrow C\left(P_{h}\right)+$ $X$, where the 4-momenta and polarizations of the incoming protons $A, B$ and outgoing hadron $C$ are specified. In twist-3 collinear QCD factorization one has

$$
\begin{aligned}
d \sigma\left(\vec{S}_{\perp}\right) & =H \otimes f_{a / A(3)} \otimes f_{b / B(2)} \otimes D_{C / c(2)} \\
& +H^{\prime} \otimes f_{a / A(2)} \otimes f_{b / B(3)} \otimes D_{C / c(2)} \\
& +H^{\prime \prime} \otimes f_{a / A(2)} \otimes f_{b / B(2)} \otimes D_{C / c(3)},
\end{aligned}
$$

with $f_{a / A(t)}\left(f_{b / B(t)}\right)$ indicating the distribution function associated with parton $a(b)$ in proton $A(B)$, while $D_{C / c(t)}$ represents the fragmentation function associated with hadron $C$ in parton $c$. The twist of the functions is denoted by $t$. The hard factors corresponding to each term are given by $H, H^{\prime}$, and $H^{\prime \prime}$, and the symbol $\otimes$ represents convolutions in the appropriate momentum fractions. In Eq. (1) a sum over partonic channels and parton flavors in each channel is understood.

The first term in (1) has already been studied in quite some detail in the literature $[5,7-12$. It contains both quark-gluon-quark correlations and tri-gluon correlations in the polarized proton, where for the former one needs to distinguish between contributions from so-called soft gluon poles (SGPs) and soft fermion poles (SFPs). The second term in (1), arising from twist-3 effects in the unpolarized proton, was shown to be small [13]. Only recently a complete analytical result was obtained for the third term in (11), which describes the twist-3 contribution due to parton fragmentation [14].

For quite some time many in the community believed that the first term in (10) dominates the transverse SSA in $p^{\uparrow} p \rightarrow h X$ (typically denoted by $A_{N}$ ) for the production of light hadrons (see, e.g., Refs. [5, 7, 10]), where the SGP contribution is generally considered the most important part. Note that the SGP contribution to $A_{N}$ is determined by the Qiu-Sterman function $T_{F}$ [4, 5], which can be related to the transverse-momentum dependent (TMD) Sivers parton density $f_{1 T}^{\perp}$ [15, 16]. For a given quark flavor $q$, these entities satisfy [17]

$$
T_{F}^{q}(x, x)=-\left.\int d^{2} \vec{p}_{\perp} \frac{\vec{p}_{\perp}^{2}}{M} f_{1 T}^{\perp q}\left(x, \vec{p}_{\perp}^{2}\right)\right|_{\mathrm{SIDIS}},
$$

where $M$ is the nucleon mass. Because of the relation in (2), one can extract $T_{F}$ from data on either $A_{N}$ or on the Sivers transverse SSA in semi-inclusive deep-inelastic scattering (SIDIS) $A_{\text {SIDIS. It }}^{\text {Siv }}$. It therefore came as a major surprise when an attempt failed to simultaneously explain both $A_{N}$ and $A_{\text {SIDIS }}^{\text {Siv }}$ [1]. The striking result pointed out in Ref. [11] was that the two extractions for $T_{F}$ differ in sign. This "sign-mismatch" puzzle could not be resolved by more flexible parameterizations of $f_{1 T}^{\perp}$ [18]. Also tri-gluon correlations are unlikely to fix 
this issue [12], while SFPs may play some role [9].

At this point one may start to question the dominance of the first term in (11). In fact, data on the transverse SSA in inclusive DIS [19, 20] seem to support this point of view, i.e., that the first term in (1) is not the main cause of $A_{N}$ [21]. Therefore, in the present work we study the potential role of the twist-3 fragmentation part of (1). After fixing the SGP contribution to $A_{N}$ through the Sivers function extracted from data on $\left.A_{\text {SIDIS }}^{\text {Siv }} 22,23\right]$ and the relation in (2), we obtain a very good fit to all high transverse-momentum forward-region pion data for $A_{N}$ from the Relativistic Heavy Ion Collider (RHIC). As explained below in more detail, our analysis shows for the first time that one can simultaneously describe $A_{N}$ using collinear factorization, $A_{\text {SIDIS }}^{\text {Siv }}$, the Collins transverse SSA $A_{\text {SIDIS }}^{\mathrm{Col}}$ in SIDIS, and $A_{e^{+} e^{-}}^{\cos (2 \phi)}$ that represents a particular azimuthal asymmetry in electron-positron annihilation into two hadrons, $e^{+} e^{-} \rightarrow h_{1} h_{2} X$ [24].

Fragmentation contribution to $A_{N}$ The fragmentation contribution to the cross section in (1) reads [14]

$$
\begin{aligned}
\frac{P_{h}^{0} d \sigma\left(\vec{S}_{\perp}\right)}{d^{3} \vec{P}_{h}}=-\frac{2 \alpha_{s}^{2} M_{h}}{S} \epsilon_{\perp, \alpha \beta} S_{\perp}^{\alpha} P_{h \perp}^{\beta} \sum_{i} \sum_{a, b, c} \int_{z_{m i n}}^{1} \frac{d z}{z^{3}} \\
\quad \times \int_{x_{\text {min }}^{\prime}}^{1} \frac{d x^{\prime}}{x^{\prime}} \frac{1}{x} \frac{1}{x^{\prime} S+T / z} \frac{1}{-x^{\prime} \hat{t}-x \hat{u}} h_{1}^{a}(x) f_{1}^{b}\left(x^{\prime}\right) \\
\quad \times\left\{\left[\hat{H}^{C / c}(z)-z \frac{d \hat{H}^{C / c}(z)}{d z}\right] S_{\hat{H}}^{i}+\frac{1}{z} H^{C / c}(z) S_{H}^{i}\right. \\
\left.+2 z^{2} \int_{z}^{\infty} \frac{d z_{1}}{z_{1}^{2}} \frac{1}{\frac{1}{z}-\frac{1}{z_{1}}} \hat{H}_{F U}^{C / c, \Im}\left(z, z_{1}\right) \frac{1}{\xi} S_{\hat{H}_{F U}}^{i}\right\},
\end{aligned}
$$

where $i$ denotes the channel, $x=-x^{\prime}(U / z) /\left(x^{\prime} S+T / z\right)$, $x_{\min }^{\prime}=-(T / z) /(U / z+S), z_{\min }=-(T+U) / S$, and $\xi=\left(1-z / z_{1}\right)$. Here we used the Mandelstam variables $S=\left(P+P^{\prime}\right)^{2}, T=\left(P-P_{h}\right)^{2}$, and $U=\left(P^{\prime}-P_{h}\right)^{2}$, which on the partonic level give $\hat{s}=x x^{\prime} S, \hat{t}=x T / z$, and $\hat{u}=x^{\prime} U / z$. Oftentimes one also uses $x_{F}=2 P_{h z} / \sqrt{S}$, where $P_{h z}$ is the longitudinal momentum of the hadron, as well as the pseudo-rapidity $\eta=-\ln \tan (\theta / 2)$, where $\theta$ is the scattering angle. The variables $x_{F}, \eta$ are further related by $x_{F}=2 P_{h \perp} \sinh (\eta) / \sqrt{S}$, where $P_{h \perp}$ is the transverse momentum of the hadron. The non-perturbative parts in (3) are the transversity distribution $h_{1}$, the unpolarized parton density $f_{1}$, and the three (twist-3) fragmentation functions (FFs) $\hat{H}, H$, and $\hat{H}_{F U}^{\Im}$, with the last one parameterizing the imaginary part of a 3-parton correlator. The definition of those functions and the results for the hard scattering coefficients $S^{i}$ can be found in [14]. (An alternative notation of the relevant FFs is given in Ref. 25], where twist-3 effects in SIDIS were computed.) We note that the so-called derivative term in (3), associated with $d \hat{H} / d z$, was first obtained in [26].

The function $\hat{H}$ is related to the TMD Collins function
$H_{1}^{\perp}$ 27] according to [14, 26]

$$
\hat{H}^{h / q}(z)=z^{2} \int d^{2} \vec{k}_{\perp} \frac{\vec{k}_{\perp}^{2}}{2 M_{h}^{2}} H_{1}^{\perp h / q}\left(z, z^{2} \vec{k}_{\perp}^{2}\right) .
$$

This relation can be considered the fragmentation counterpart of Eq. (2). Exploiting the universality of the Collins function 28, one can simultaneously extract $H_{1}^{\perp}$ and $h_{1}$ from data on $A_{\text {SIDIS }}^{\text {Col }}$ 29, 30] and data on $A_{e^{+} e^{-}}^{\cos (2 \phi)}$ [31, 32] (see [33] and references therein). Below we utilize such information for $H_{1}^{\perp}$ and $h_{1}$ when describing $A_{N}$. The FFs in (3) are related via [14]

$$
H^{h / q}(z)=-2 z \hat{H}^{h / q}(z)+2 z^{3} \int_{z}^{\infty} \frac{d z_{1} 1}{z_{1}^{2} \frac{1}{z}-\frac{1}{z_{1}}} \hat{H}_{F U}^{h / q, \Im}\left(z, z_{1}\right),
$$

implying that in the collinear twist-3 framework one has two independent FFs. It is important to realize that this is different from the so-called TMD approach for $A_{N}$, where only $H_{1}^{\perp}$ enters the fragmentation piece [34].

Phenomenology of $A_{N}$ for pion production We consider $A_{N}$ for $p^{\uparrow} p \rightarrow \pi X$ in the forward region of the polarized proton, which has been studied by the STAR [35 37], BRAHMS 38, 39] and PHENIX [40] collaborations at RHIC. We mainly focus on data taken at $\sqrt{S}=200 \mathrm{GeV}$ for which typically $P_{h \perp}>1 \mathrm{GeV}$. Throughout we use the GRV98 unpolarized parton distributions [41] and the DSS unpolarized FFs [42]. Note that the GRV98 parton distributions were also used in Refs. [22, 23, 33] for extracting the Sivers function and the transversity, which we take as input in our calculation. The SGP contribution to (1) is computed by fixing $T_{F}$ through Eq. (2) with two different inputs for the Sivers function - SV1: $f_{1 T}^{\perp}$ from Ref. [22], obtained from SIDIS data on $A_{\text {SIDIS }}^{\text {Siv }}$ 43, 44]; and SV2: $f_{1 T}^{\perp}$ from Ref. [23], "constructed" such that, in the TMD approach, the contribution of the Sivers effect to $A_{N}$ is maximized while maintaining a good description of $A_{\text {SIDIS }}^{\text {Siv }}$. These two inputs for $f_{1 T}^{\perp}$ are mainly distinct by their quite different large- $x$ behavior. To compute the contribution in (3) we take $h_{1}$ and $H_{1}^{\perp}$ (which fixes $\hat{H}$ through (44) from [33]. For favored fragmentation into $\pi^{+}$we make for $\hat{H}_{F U}^{\Im}$ the ansatz

$$
\begin{array}{r}
\frac{\hat{H}_{F U}^{\pi^{+} /(u, \bar{d}), \Im}\left(z, z_{1}\right)}{D^{\pi^{+} /(u, \bar{d})}(z) D^{\pi^{+} /(u, \bar{d})}\left(z / z_{1}\right)}=\frac{N_{\mathrm{fav}}}{2 I_{\mathrm{fav}} J_{\mathrm{fav}}} z^{\alpha_{\mathrm{fav}}}\left(z / z_{1}\right)^{\alpha_{\mathrm{fav}}^{\prime}} \\
\times(1-z)^{\beta_{\mathrm{fav}}}\left(1-z / z_{1}\right)^{\beta_{\mathrm{fav}}^{\prime}}, \quad(6)
\end{array}
$$

with the parameters $N_{\mathrm{fav}}, \alpha_{\mathrm{fav}}, \alpha_{\mathrm{fav}}^{\prime}, \beta_{\mathrm{fav}}, \beta_{\mathrm{fav}}^{\prime}$ and the unpolarized FF $D$. Note that the allowed range for $z$ and $z / z_{1}$ is [0,1] [45] and that our ansatz satisfies the constraint $\hat{H}_{F U}(z, z)=0$ [45, 46]. With the use of DSS FFs [42], the factor $I_{\text {fav }}$ reads $I_{\text {fav }} \equiv I_{u+\bar{u}}-I_{\bar{u}}$ where $I_{i}$ 

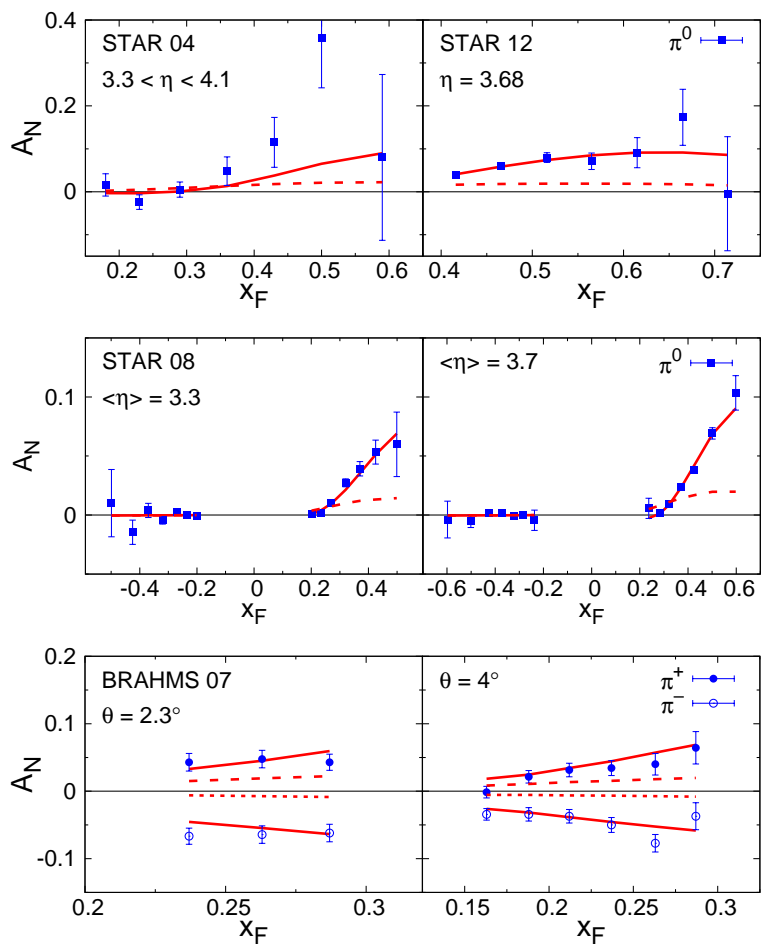

FIG. 1. Fit results for $A_{N}^{\pi^{0}}$ (data from $35-37$ ) and $A_{N}^{\pi^{ \pm}}$(data from [38]) for the SV1 input. The dashed line (dotted line in the case of $\pi^{-}$) means $\hat{H}_{F U}^{\Im}$ switched off.

$(i=u+\bar{u}, \bar{u})$ is defined as

$$
\begin{gathered}
I_{i}=\frac{N_{i}\left(K_{1, \mathrm{fav}}+\gamma_{i} K_{2, \mathrm{fav}}\right)}{B\left[2+\alpha_{i}, \beta_{i}+1\right]+\gamma_{i} B\left[2+\alpha_{i}, \beta_{i}+\delta_{i}+1\right]}, \\
\text { with } K_{1, \mathrm{fav}}=B\left[\alpha_{\mathrm{fav}}^{\prime}+\alpha_{i}+1, \beta_{\mathrm{fav}}^{\prime}+\beta_{i}\right], \\
K_{2, \mathrm{fav}}=B\left[\alpha_{\mathrm{fav}}^{\prime}+\alpha_{i}+1, \beta_{\mathrm{fav}}^{\prime}+\beta_{i}+\delta_{i}\right],
\end{gathered}
$$

and $B[a, b]$ the Euler $\beta$-function. The parameters $N_{i}$, $\alpha_{i}, \beta_{i}, \gamma_{i}$, and $\delta_{i}$ come from $D$ FFs at the initial scale and are given in Table III of [42]. Note that $D^{\pi^{+}} / u$ in Ref. [42] differs from $D^{\pi^{+} / \bar{d}}$. $J_{\text {fav }}$ in (6) is similarly defined as $J_{\text {fav }} \equiv J_{u+\bar{u}}-J_{\bar{u}}$, where $J_{i}(i=u+\bar{u}, \bar{u})$ follows from $I_{i}$ through $\alpha_{\text {fav }}^{\prime} \rightarrow\left(\alpha_{\text {fav }}+4\right), \beta_{\text {fav }}^{\prime} \rightarrow\left(\beta_{\text {fav }}+1\right)$. The factor $1 /\left(2 I_{\mathrm{fav}} J_{\mathrm{fav}}\right)$ in (6) is convenient and implies $\int_{0}^{1} d z z H_{(3)}^{\pi^{+} / u}(z)=N_{\text {fav }}$ at the initial scale, where $H_{(3)}$ represents the entire second term on the r.h.s. of (5). For the disfavored FFs $\hat{H}_{F U}^{\pi^{+} /(d, \bar{u}), \Im}$ we make an ansatz in full analogy to (6), introducing the additional parameters $N_{\mathrm{dis}}, \alpha_{\mathrm{dis}}, \alpha_{\mathrm{dis}}^{\prime}, \beta_{\mathrm{dis}}, \beta_{\mathrm{dis}}^{\prime} .\left(I_{\mathrm{dis}}\right.$ and $J_{\mathrm{dis}}$ are calculated using $D^{\pi^{+} / d}=D^{\pi^{+} / \bar{u}}$ from 42$]$.) The $\pi^{-}$FFs are then fixed through charge conjugation, and the $\pi^{0} \mathrm{FFs}$ are given by the average of the FFs for $\pi^{+}$and $\pi^{-}$. The FFs $H^{\pi / q}$ are computed by means of (5). All parton correlation functions are evaluated at the scale $P_{h \perp}$ with leading order evolution of the collinear functions.

Using the MINUIT package we fit the fragmentation contribution to data for $A_{N}^{\pi^{0}}\left[\begin{array}{ll}35 & 37\end{array}\right]$ and $A_{N}^{\pi^{ \pm}}$[38]. To facilitate the fit we only keep 7 parameters in $\hat{H}_{F U}^{\pi^{+} / q, \Im}$ free.

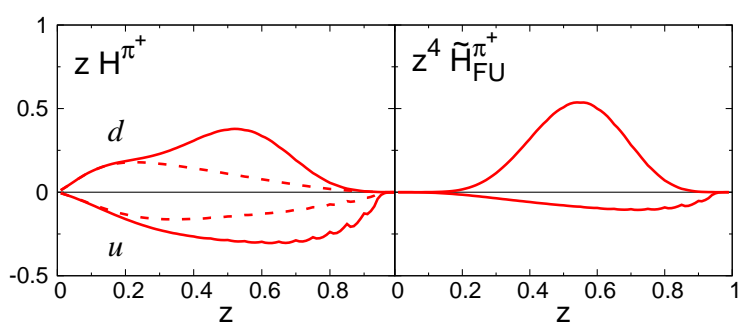

FIG. 2. Results for the FFs $H^{\pi^{+} / q}$ and $\tilde{H}_{F U}^{\pi^{+} / q}$ (defined in the text) for the SV1 input. Also shown is $H^{\pi^{+} / q}$ without the contribution from $\hat{H}_{F U}^{\Im}$ (dashed line).

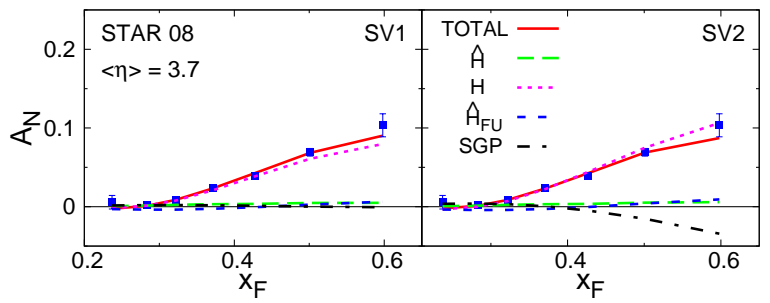

FIG. 3. Individual contributions to $A_{N}^{\pi^{0}}$ (data from [36]) for SV1 and SV2 inputs.

We also allow the $\beta$-parameters $\beta_{u}^{T}=\beta_{d}^{T}$ of the transversity to vary within the error range given in [33]. All integrations are done using the Gauss-Legendre method with 250 steps. For the SV1 input the result of our 8parameter fit is shown in Tab. [1 Note that the values for $\beta_{\mathrm{fav}}^{\prime}=\beta_{\mathrm{dis}}^{\prime}$ and $\beta_{\mathrm{fav}}$ are at their lower limits, which we introduce to guarantee a finite integration upon $z_{1}$ in (3) and a proper behavior of $A_{N}$ at large $x_{F}$, respectively. For the SV2 input the values of the fit parameters are similar, with an equally successful fit $\left(\chi^{2} /\right.$ d.o.f. $\left.=1.10\right)$.

TABLE I. Fit parameters for SV1 input.

\begin{tabular}{ll}
\hline \hline \multicolumn{2}{c}{$\chi^{2} /$ d.o.f. $=1.03$} \\
\hline$N_{\text {fav }}=-0.0338$ & $N_{\text {dis }}=0.216$ \\
$\alpha_{\text {fav }}=\alpha_{\text {fav }}^{\prime}=-0.198$ & $\beta_{\text {fav }}=0.0$ \\
$\beta_{\text {fav }}^{\prime}=\beta_{\text {dis }}^{\prime}=-0.180$ & $\alpha_{\text {dis }}=\alpha_{\text {dis }}^{\prime}=3.99$ \\
$\beta_{\text {dis }}=3.34$ & $\beta_{u}^{T}=\beta_{d}^{T}=1.10$ \\
\hline \hline
\end{tabular}

The very good description of $A_{N}$ is also reflected by Fig. 1. We emphasize that such a positive outcome is non-trivial if one keeps in mind the constraint in (5) and the need to simultaneously fit data for $A_{N}^{\pi^{0}}$ and $A_{N}^{\pi^{ \pm}}$. Results for the FFs $H^{\pi^{+} / q}$ and $\tilde{H}_{F U}^{\pi^{+} / q} \equiv$ $\int_{z}^{\infty} \frac{d z_{1}}{z_{1}^{2}} \frac{1}{\frac{1}{z}-\frac{1}{z_{1}}} \frac{1}{\xi} \hat{H}_{F U}^{\pi^{+} / q, \Im}\left(z, z_{1}\right)$ are displayed in Fig. 2. In either case the favored and disfavored FFs have opposite signs. This is like for $H_{1}^{\perp}$ where such reversed signs are actually "preferred" by the Schäfer-Teryaev (ST) sum rule $\sum_{h} \sum_{S_{h}} \int_{0}^{1} d z z M_{h} \hat{H}^{h / q}(z)=0$ [47]. Note that the ST sum rule, in combination with (5), implies a constraint on a certain linear combination of $H^{h / q}$ and (an 


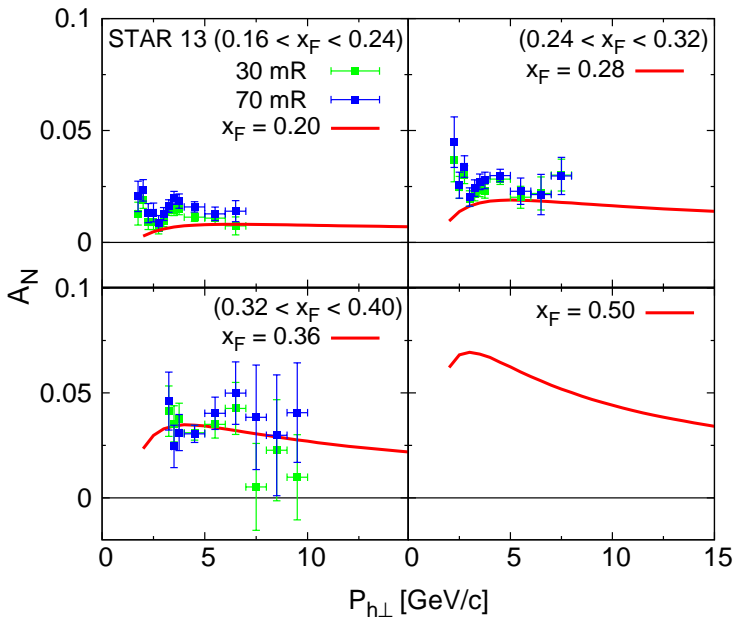

FIG. 4. $A_{N}$ as function of $P_{h \perp}$ for SV1 input at $\sqrt{S}=$ $500 \mathrm{GeV}$ (data from [48]).

integral of) $\hat{H}_{F U}^{h / q, \Im}$. In view of that, reversed signs between favored and disfavored FFs like in Fig. 2 are actually beneficial. Also depicted in Fig. 2 is $H^{\pi^{+} / q}$ when $\hat{H}_{F U}^{\pi^{+} / q, \Im}$ is switched off. As shown in Fig. 1] in such a scenario, i.e., by turning off the 3-parton FF, one cannot describe the data for $A_{N}$. According to Fig. 3, the $\hat{H}$ term (including its derivative) in (3) contributes only very little to $A_{N}$. Also the SGP pole term is small, except for the SV2 input at large $x_{F}$, where its contribution is opposite to the data. Clearly $A_{N}$ is governed by the $H$-term in (3). This result can mainly be traced back to the hard scattering coefficients: e.g., for the dominant $q g \rightarrow q g$ channel one has $S_{H} \propto 1 / \hat{t}^{3}$, but $S_{\hat{H}} \propto 1 / \hat{t}^{2}[14]$ in the forward region where $\hat{t}$ is small. Finally, Fig. 4 shows the $P_{h \perp}$-dependence of $A_{N}$ for $\sqrt{S}=500 \mathrm{GeV}$. Preliminary data from STAR, extending to almost $P_{h \perp}=10 \mathrm{GeV}$, show that $A_{N}$ is rather flat [48]. The twist-3 calculation agrees with that trend, and also the magnitude of $A_{N}$ is in line with the data. Note that the data of Ref. [48] were not included in our fit and that only statistical errors are shown in Fig. 4 48.

Conclusions Collinear twist-3 QCD factorization can be considered the most natural and rigorous approach to the transverse SSA $A_{N}$ in $p^{\uparrow} p \rightarrow h X$. However, the signmismatch issue of the Sivers effect had put this framework into question [11]. Here we have demonstrated for the first time that, despite the sign-mismatch problem, twist-3 factorization actually can describe high-energy RHIC data for $A_{N}^{\pi}$ very well if one takes the fragmentation contribution into account. We re-emphasize that this result is non-trivial. Since in the twist-3 approach part of $A_{N}$ can be fixed by spin/azimuthal asymmetries in SIDIS and in $e^{+} e^{-} \rightarrow h_{1} h_{2} X$, we have shown that at present a simultaneous description of all those observables is possible. We repeat that the fragmentation con- tribution in twist-3 factorization goes beyond the pure Collins effect. Independent information on the FFs $H^{\pi / q}$, $\hat{H}_{F U}^{\pi / q, \Im}$ from other sources is needed before one can ultimately claim the intriguing data on $A_{N}^{\pi}$ is fully understood. However, the fact that $\hat{H}_{F U}^{\pi / q, \Im}$ gives a reasonable contribution to (the numerically dominant) $H^{\pi / q}$ (see Fig. 2) allows one to be optimistic in this regard.

We thank S. Heppelmann, E.-C. Aschenauer, and R. Fatemi for their effort in making the data of Ref. [48] available to us. We also thank S. Scopetta, W. Vogelsang, and S. Yoshida for support with regards to the evolution of the transversity distribution. This work has been supported by the Grant-in-Aid for Scientific Research from the Japanese Society of Promotion of Science under Contract Nos. 24.6959 (K.K.), 23540292 and 26287040 (Y.K.), the National Science Foundation under Contract No. PHY-1205942 (A.M.), and the RIKEN BNL Research Center (D.P.).

[1] G. Bunce et al., Phys. Rev. Lett. 36, 1113 (1976).

[2] G. L. Kane, J. Pumplin and W. Repko, Phys. Rev. Lett. 41, 1689 (1978).

[3] A. V. Efremov and O. V. Teryaev, Sov. J. Nucl. Phys. 36, 140 (1982) [Yad. Fiz. 36, 242 (1982)]; Phys. Lett. B 150, 383 (1985).

[4] J.-w. Qiu and G. F. Sterman, Phys. Rev. Lett. 67, 2264 (1991); Nucl. Phys. B 378, 52 (1992).

[5] J.-w. Qiu and G. F. Sterman, Phys. Rev. D 59, 014004 (1999).

[6] H. Eguchi, Y. Koike and K. Tanaka, Nucl. Phys. B 752, 1 (2006); Nucl. Phys. B 763, 198 (2007).

[7] C. Kouvaris et al., Phys. Rev. D 74, 114013 (2006).

[8] Y. Koike and K. Tanaka, Phys. Rev. D 76, 011502 (2007).

[9] Y. Koike and T. Tomita, Phys. Lett. B 675, 181 (2009).

[10] K. Kanazawa and Y. Koike, Phys. Rev. D 82, 034009 (2010); Phys. Rev. D 83, 114024 (2011).

[11] Z.-B. Kang et al., Phys. Rev. D 83, 094001 (2011).

[12] H. Beppu et al., Phys. Rev. D 89, 034029 (2014).

[13] Y. Kanazawa and Y. Koike, Phys. Lett. B 478, 121 (2000); Phys. Lett. B 490, 99 (2000).

[14] A. Metz and D. Pitonyak, Phys. Lett. B 723, 365 (2013).

[15] D. W. Sivers, Phys. Rev. D 41, 83 (1990); Phys. Rev. D 43, 261 (1991).

[16] D. Boer and P. J. Mulders, Phys. Rev. D 57, 5780 (1998).

[17] D. Boer, P. J. Mulders and F. Pijlman, Nucl. Phys. B 667, 201 (2003).

[18] Z.-B. Kang and A. Prokudin, Phys. Rev. D 85, 074008 (2012).

[19] A. Airapetian et al. [HERMES Collaboration], Phys. Lett. B 682, 351 (2010).

[20] J. Katich et al., arXiv:1311.0197 [nucl-ex].

[21] A. Metz et al., Phys. Rev. D 86, 094039 (2012).

[22] M. Anselmino et al., Eur. Phys. J. A 39, 89 (2009).

[23] M. Anselmino et al., Phys. Rev. D 88, 054023 (2013).

[24] D. Boer, R. Jakob and P. J. Mulders, Nucl. Phys. B 504, 345 (1997); D. Pitonyak, M. Schlegel and A. Metz, arXiv:1310.6240 [hep-ph]. 
[25] K. Kanazawa and Y. Koike, Phys. Rev. D 88, 074022 (2013).

[26] Z.-B. Kang, F. Yuan and J. Zhou, Phys. Lett. B 691, $243(2010)$.

[27] J. C. Collins, Nucl. Phys. B 396, 161 (1993).

[28] A. Metz, Phys. Lett. B 549, 139 (2002); J. C. Collins and A. Metz, Phys. Rev. Lett. 93, 252001 (2004).

[29] A. Airapetian et al. [HERMES Collaboration], Phys. Lett. B 693, 11 (2010).

[30] C. Adolph et al. [COMPASS Collaboration], Phys. Lett. B 717, 376 (2012).

[31] R. Seidl et al. [Belle Collaboration], Phys. Rev. D 78, 032011 (2008) [Erratum-ibid. D 86, 039905 (2012)].

[32] J. P. Lees et al. [BaBar Collaboration], arXiv:1309.5278 [hep-ex].

[33] M. Anselmino et al., Phys. Rev. D 87, 094019 (2013).

[34] M. Anselmino et al., Phys. Rev. D 86, 074032 (2012).

[35] J. Adams et al. [STAR Collaboration], Phys. Rev. Lett. 92, 171801 (2004).

[36] B. I. Abelev et al. [STAR Collaboration], Phys. Rev. Lett. 101, 222001 (2008).

[37] L. Adamczyk et al. [STAR Collaboration], Phys. Rev. D 86, 051101 (2012).

[38] J. H. Lee et al. [BRAHMS Collaboration], AIP Conf.
Proc. 915, 533 (2007).

[39] I. Arsene et al. [BRAHMS Collaboration], Phys. Rev. Lett. 101, 042001 (2008).

[40] A. Adare et al. [PHENIX Collaboration], arXiv:1312.1995 [hep-ex].

[41] M. Glück, E. Reya and A. Vogt, Eur. Phys. J. C 5, 461 (1998).

[42] D. de Florian, R. Sassot and M. Stratmann, Phys. Rev. D 75, 114010 (2007).

[43] M. Alekseev et al. [COMPASS Collaboration], Phys. Lett. B 673, 127 (2009).

[44] A. Airapetian et al. [HERMES Collaboration], Phys. Rev. Lett. 103, 152002 (2009).

[45] S. Meissner and A. Metz, Phys. Rev. Lett. 102, 172003 (2009).

[46] L. P. Gamberg, A. Mukherjee and P. J. Mulders, Phys. Rev. D 77, 114026 (2008); Phys. Rev. D 83, 071503 (2011).

[47] A. Schäfer and O. V. Teryaev, Phys. Rev. D 61, 077903 (2000); S. Meissner, A. Metz and D. Pitonyak, Phys. Lett. B 690, 296 (2010).

[48] S. Heppelmann [STAR Collaboration], PoS DIS 2013, 240 (2013). 